\title{
Modulation of inflammation in wounds of diabetic patients treated with porcine urinary bladder matrix
}

\author{
John T Paige1, Michael Kremer², Jace Landry¹, Samuel A Hatfield², Donald Wathieu², \\ Aaron Brug ${ }^{2}$, Daniel J Lightell ${ }^{2}$, Kara L Spiller ${ }^{3}$ \& T Cooper Woods ${ }^{*, 2}$ \\ ${ }^{1}$ Department of Surgery, LSU Health New Orleans, School of Medicine, New Orleans, LA 70112, USA \\ ${ }^{2}$ Departments of Physiology \& Medicine, Tulane School of Medicine, New Orleans, LA 70112, USA \\ ${ }^{3}$ School of Biomedical Engineering Science \& Health Systems, Drexel University, Philadelphia, 19104 PA, USA \\ *Author for correspondence: Tel.: +1 504988 2588; Twoods3@tulane.edu
}

\begin{abstract}
Aim: To determine if porcine urinary bladder matrix (UBM) treatment is associated with modulation of wound inflammation in diabetic patients. Patients \& methods: mRNA associated with M1 and M2 macrophages were measured in wounds of diabetic and nondiabetic patients pre- and post-treatment with UBM and an M1:M2 score was calculated. Results: Wound tissue from diabetic subjects exhibited elevated M1:M2 scores compared with nondiabetic patients, suggesting a greater pro-inflammatory state prior to treatment. Post-treatment, there was significantly greater reduction in the magnitude of the individual M1:M2 scores in the diabetic patients resulting in similar levels in both groups of patients. Conclusions: UBM may assist in diabetic wound healing by restoring an inflammatory state similar to that of nondiabetic patients.
\end{abstract}

First draft submitted: 18 January 2019; Accepted for publication: 20 March 2019; Published online: 25 April 2019

Keywords: acellular xenograft $\bullet$ biomaterials $\bullet$ diabetes mellitus $\bullet$ macrophage polarization $\bullet$ skin and wound care - wound healing

Diabetes mellitus is a debilitating chronic disease that places a large financial burden on the US healthcare system. In 2012 alone, diabetes accounted for $\$ 176$ billion in direct medical costs and $\$ 69$ billion in reduced productivity [1]. One manner in which such costs are generated is through the management of chronic wounds that arise and persist in diabetics due to impairment of the normal wound healing process.

Macrophages are key mediators of proper wound healing that exist in multiple phenotypes with diverse functionality [2]. Normal wound healing begins with an early inflammatory stage dominated by the pro-inflammatory M1 macrophage. This macrophage phenotype secretes pro-inflammatory cytokines and serves to clear pathogens and damaged tissue. Over time, a phenotypic switch occurs toward the M2 phenotype that promotes wound healing and decreases inflammation. Recently, Nassiri et al. reported that the relative level of inflammation of a wound can be monitored by measuring a set of mRNA associated with the M1 (VEGF, CCR7, IL1 $\beta, C D 80)$ and M2 (MRC1, TIMP3, PDGF) macrophage phenotypes and calculating a M1:M2 score [3]. In diabetic subjects, the ratio of M1 (pro-inflammatory) to M2 (anti-inflammatory) macrophages is increased [4-7]. As a result, the proliferative phase is impeded in diabetic subjects, delaying wound healing.

Urinary bladder matrix (UBM) is an extracellular matrix scaffold derived from the mucosa of porcine urinary bladder that provides a structural scaffold for cell migration, adhesion and proliferation [8-10]. UBM has been shown to foster a proportional increase in M2 macrophages both in vitro and in animal models [7,11,12]. As such, macrophages in diabetic patients may reverse their abnormal phenotypic behavior, thereby promoting a normal healing response.

The use of UBM for the management of chronic wounds in diabetic patients has been described in numerous case reports and two prospective randomized trials with promising results [13-16]. The aim of this study was to test the ability of UBM to affect macrophage phenotype-related gene expression and the resultant M1:M2 score in the wounds of diabetic subjects compared to that observed in nondiabetic patients. Using wound debridement samples 
and the M1:M2 score, we examined M1:M2 content pre- and post-UBM application in diabetic and nondiabetic patients. These data provide initial mechanistic insight underlying the ability of UBM to facilitate proper wound healing in the diabetic setting.

\section{Patients \& methods}

\section{Patient population \& wound debridement}

This single center, prospective case-control study was conducted at University Medical Center New Orleans (UMCNO) from November 2015 to April 2017. Wound measurement, debridement and UBM application were performed by a single general surgeon physician. Included participants were English speaking consecutive patients aged 18-65 years with open wounds that had not previously been treated with a biologically derived scaffold referred to the UMCNO Wound Care Clinic for wound management after the study start date who consented to receiving the UBM product. Exclusion criteria were clinically infected wounds (i.e., wounds demonstrating Galen's dolor, calor, tumor and rubor), concurrent hyperbaric therapy, non-English speaking patients, prior therapy of the target wound with biologically derived products (i.e., UBM, Oasis, Primatrix, etc.) and patients $<18$ or $>65$ years. Informed consent was obtained from each patient and the study was approved by both the Louisiana State University (LSU) Health New Orleans Health Sciences Center and the UMCNO Institutional Review Boards. All patients provided written informed consent to participate in the study.

Patients were referred to the UMCNO Wound Care Clinic from inpatient discharge referrals with initial accrual of 20 patients (10 diabetic and 10 nondiabetic). Two patients, however, chose to withdraw and these data were not included. Wound debridement samples along with appropriate medical history were obtained from nondiabetic and diabetic patients, immediately prior to application of UBM. Upon completion of debridement, Cytal ${ }^{\circledR}$ Wound Matrix 1-Layer (single layer sheet, ACell, Inc., MD, USA), MicroMatrix ${ }^{\circledR}$ (particulate, ACell, Inc.) or both UBM products were applied throughout each wound according to depth and size, and they were covered with a waterbased lubricant, nonadherent dressing and final securing over dressing. A second wound debridement sample was obtained 7-14 days after UBM application. The second sample was not obtained from two nondiabetic patients due to failure to collect the sample and one diabetic patient due to wound healing. Wound area reduction was measured at the time of the second debridement sample collection. Pretreatment wound area reduction was calculated from data in the patients' medical history. The rate of wound area reduction was calculated as the percent decrease in wound size divided by the number of days between wound measurements. Hemoglobin A1c values measured pretreatment and within 3 months of the end of treatment were obtained from the medical records of a subset of the diabetic patients $(n=6)$.

\section{RNA measurement}

Debridement samples were stored in RNAlater solution until total RNA was isolated using the Qiagen MiniKit (Qiagen, Hilden, Germany). Copies of mRNA associated with the M1 (VEGF, CCR7, IL1 $\beta, C D 80)$ and M2 (MRC1, TIMP3, PDGF) macrophage phenotype were measured in the samples using droplet digital PCR (ddPCR). A one-step PCR reaction was prepared using Qiagen primers (Supplementary Table 1) coupled with Superscript reverse transcriptase (Qiagen) and the QX200 ddPCR EvaGreen Supermix (Bio-Rad, CA, USA). Reaction droplets were prepared and read using the QX200 ddPCR system.

\section{Data analysis}

Using the methodology of Nassiri et al., we calculated an M1:M2 score that is higher for M1 macrophages and lower for M2 macrophages, and that has been shown to increase in nonhealing wounds and decrease in healing wounds $[3,17]$. Prior to calculating the M1:M2 score, the gene expression data were normalized to prevent bias due to differing baseline gene expression. The copy numbers of each individual mRNA were normalized by dividing them by the median of copy number for that mRNA. The M1:M2 score was calculated as the ratio of the sum of the normalized values of M1 associated mRNAs to the sum of the normalized values for the M2 associated mRNA using the following equation:

$$
\text { M1:M2 Score }=\frac{\left(X_{V E G F}+X_{C C R 7}+X_{I L 1 \beta}+X_{C D 80}\right)}{\left(X_{P D G F}+X_{M R C 1}+X_{T I M P 3}\right)}
$$


Table 1. Demographic and clinical variables of study patients.

\begin{tabular}{|lllll|}
\hline Characteristics & All patients & Nondiabetic $(\mathrm{n}=9)$ & Diabetic $(\mathrm{n}=9)$ & p-value \\
\hline Age, years & $51.8 \pm 2.6$ & $46.8 \pm 4.7$ & $55.8 \pm 1.3$ & 0.13 \\
\hline BMI, lb/in ${ }^{2}$ & $35.1 \pm 1.8$ & $32.5 \pm 2.4$ & $37.6 \pm 2.6$ & 0.18 \\
\hline Initial wound area, $\mathrm{cm}^{2}$ & $6.5 \pm 1.7$ & $3.8 \pm 1.2$ & $8.3 \pm 2.9$ & 0.25 \\
\hline Male sex & $9(50)$ & $5(56)$ & $4(44)$ & 0.64 \\
\hline Smoker & $13(72)$ & $7(39)$ & $6(33)$ & 1 \\
\hline
\end{tabular}

$X_{\text {gene }}=\frac{\text { copies of gene in sample }}{\text { median of copies of the gene in all samples }}$

\section{Statistical analysis}

Comparison of continuous variable means between nondiabetic and diabetic patients was performed using paired Student's t-test. Differences in categorical variables were determined using $\chi^{2}$ analysis. A p $<0.05$ was considered statistically significant.

\section{Data availability}

The datasets generated during and/or analyzed during the current study are available from the corresponding author on reasonable request.

\section{Results}

\section{Patient population}

A total of nine diabetic and nine nondiabetic patient samples were analyzed. Within this group, mean patient age was $51.8 \pm 2.6$ years (range: $20-62)$ and $50 \%\left(\mathrm{n}=9\right.$ ) were male. Mean BMI was $35.1 \pm 1.8 \mathrm{lb} /$ in $^{2}$. Hemoglobin A1c values in the diabetic patients demonstrated improved glucose control during the treatment period compared with prior to treatment with mean values of $8.0 \pm 0.7$ versus $10.3 \pm 1.0$, respectively. The average initial wound area was $6.5 \pm 1.7 \mathrm{~cm}^{2}$. More than $70 \%(\mathrm{n}=13)$ of the patients in this study were smokers. No significant differences were found in age, BMI, initial wound area or smoking status between the diabetic and nondiabetic groups (Table 1). The duration of wounds prior to treatment is listed in Table 2. Wounds were located on the foot $(n=9)$, abdomen $(n=5)$, hand $(n=2)$, hip, head and thigh. Most wounds were those that developed after a patient had undergone an operative procedure to treat a surgical condition $(n=12)$. Application of UBM was associated with significant wound size reduction in both patient groups with a $35 \% \pm 14 \%$ reduction in the diabetic and a $43 \% \pm 18 \%$ reduction in the nondiabetic patients $(\mathrm{p}<0.05$, Figure $1 \mathrm{~A})$, with all but one diabetic patient exhibiting some magnitude of wound closure. A significant increase in the rate of wound area reduction compared with pretreatment was also observed in both patient groups (Figure $1 \mathrm{~B} ; \mathrm{p}<0.05$ ). One diabetic patient's wound fully healed prior to the follow-up visit and therefore only a pretreatment sample was obtained.

\section{Macrophage phenotype associated mRNA}

Wound healing is associated with a transition of macrophages from the M1 to M2 phenotype, and wounds in diabetic patients are known to exhibit elevated levels of M1 macrophages compared with M2. To assess the character of the inflammation in the wounds, we measured a set of mRNAs associated with the M1 (IL1 $\beta$, CD80, CCR7 and VEGF) and M2 (PDGF, MRC1 and TIMP3) phenotypes pre- and post-treatment. In the total population, significant increases in VEGF, MRC1 and TIMP3 were observed following UBM treatment (Figure 2A). Similar trends were observed in the diabetic and nondiabetic groups individually, although significance was only found in TIMP3 in the nondiabetic population (Figure 2B) and VEGF in the diabetic population (Figure 2C).

\section{M1:M2 scores}

A score based on the ratio of the expression of the genes associated with the macrophage phenotypes (M1:M2 score) has been previously demonstrated to be indicative of wound healing and the character of the wound inflammation [3,17]. The diabetic patients in this study exhibited a significantly higher M1:M2 score pretreatment compared with post-treatment ( $4.42 \pm 0.58$ vs $2.075 \pm 0.94 ; \mathrm{p}<0.05$; Figure $3 \mathrm{~A})$. The M1:M2 score was reduced to similar levels in both diabetic and nondiabetic patients following UBM treatment $(2.06 \pm 0.74$ vs $1.67 \pm 0.75$; 


\begin{tabular}{|c|c|c|c|c|}
\hline Patient & Diabetic & Etiology & Location & $\begin{array}{l}\text { Duration prior to treatment } \\
\text { (days) }\end{array}$ \\
\hline 1 & $\mathrm{~N}$ & Surgical & Abdomen & 58 \\
\hline 2 & $\mathrm{Y}$ & Surgical & Foot & 63 \\
\hline 3 & $\mathrm{~N}$ & Surgical & Hand & 14 \\
\hline 4 & $\mathrm{Y}$ & Diabetic foot ulcer & Foot & 219 \\
\hline 7 & $\mathrm{~N}$ & Surgical & Hip & 12 \\
\hline 8 & $\mathrm{Y}$ & Surgical & Foot & 59 \\
\hline 9 & $\mathrm{~N}$ & Venous stasis ulcer & Foot & 292 \\
\hline 10 & Y & Surgical & Abdomen & 14 \\
\hline 15 & Y & Diabetic foot ulcer & Foot & 636 \\
\hline 16 & $\mathrm{~N}$ & Surgical & Back & 16 \\
\hline 17 & $\mathrm{~N}$ & Laceration & Head & 45 \\
\hline 18 & $\mathrm{~N}$ & Surgical & Thigh & 35 \\
\hline
\end{tabular}

(A)

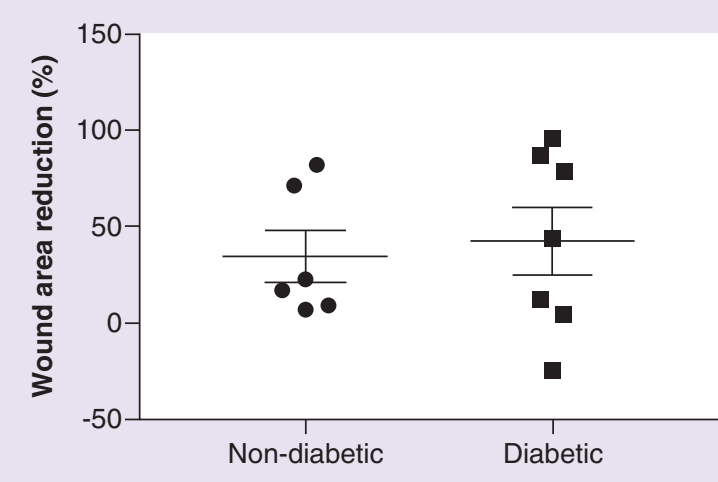

(B)

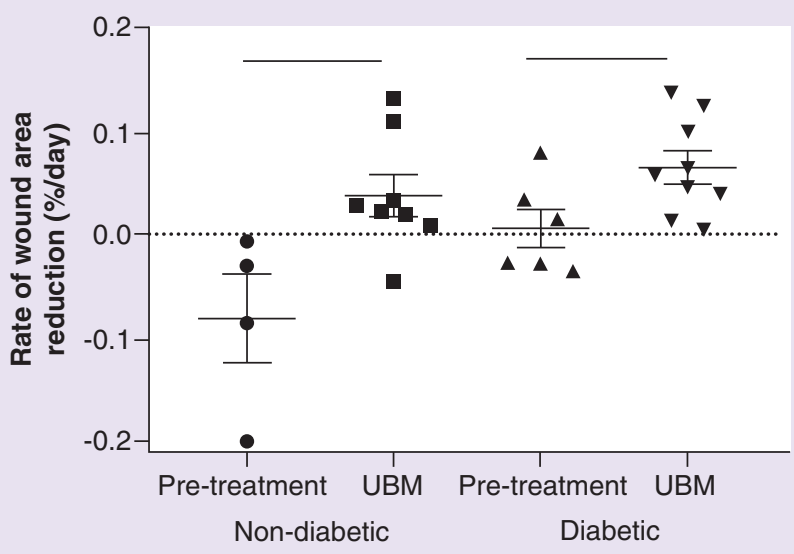

Figure 1. Comparison of wound area reduction in diabetic and nondiabetic patients. (A) Similar wound area reduction was observed in the diabetic and nondiabetic patient groups. (B) UBM was associated with a significant increase in the rate of wound area closure compared with pretreatment in both the diabetic and nondiabetic patient groups. Bar indicates $p<0.05$.

UBM: Urinary bladder matrix.

$\mathrm{p}=0.36$ ). This represents a significantly greater decrease in the magnitude of change in the individual M1:M2 scores in the diabetic versus nondiabetic patients $(2.89 \pm 0.68$ vs $0.66 \pm 0.53 ; \mathrm{p}<0.05)$. Additionally, the magnitude of the decrease in the M1:M2 score correlated with the magnitude of the rate of wound area reduction $\left(R^{2}=0.36\right.$; $\mathrm{p}=0.04$; Figure $3 \mathrm{~B}$ ). These data suggest UBM treatment is effective at restoring the state of inflammation in the wounds of diabetic patients to that of nondiabetic patients.

\section{Discussion}

In this study, we use a score to characterize the inflammation of wounds before and after treatment with UBM. Prior to treatment, the wounds of diabetic patients exhibited a significantly higher M1:M2 score, suggesting greater 
(A)

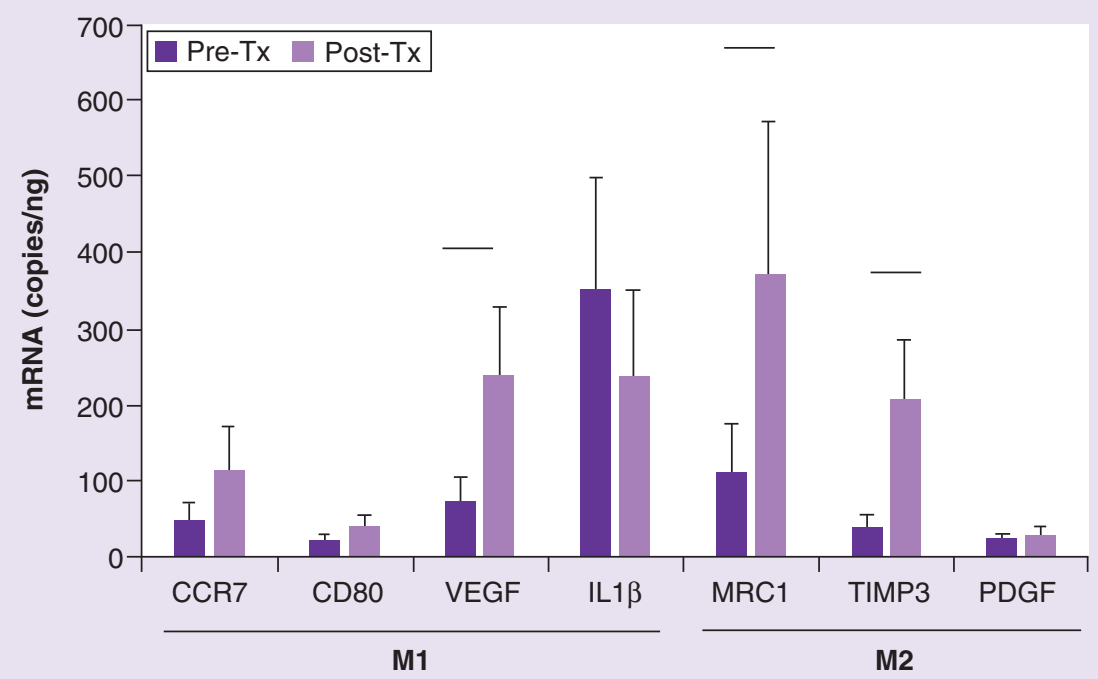

(B)

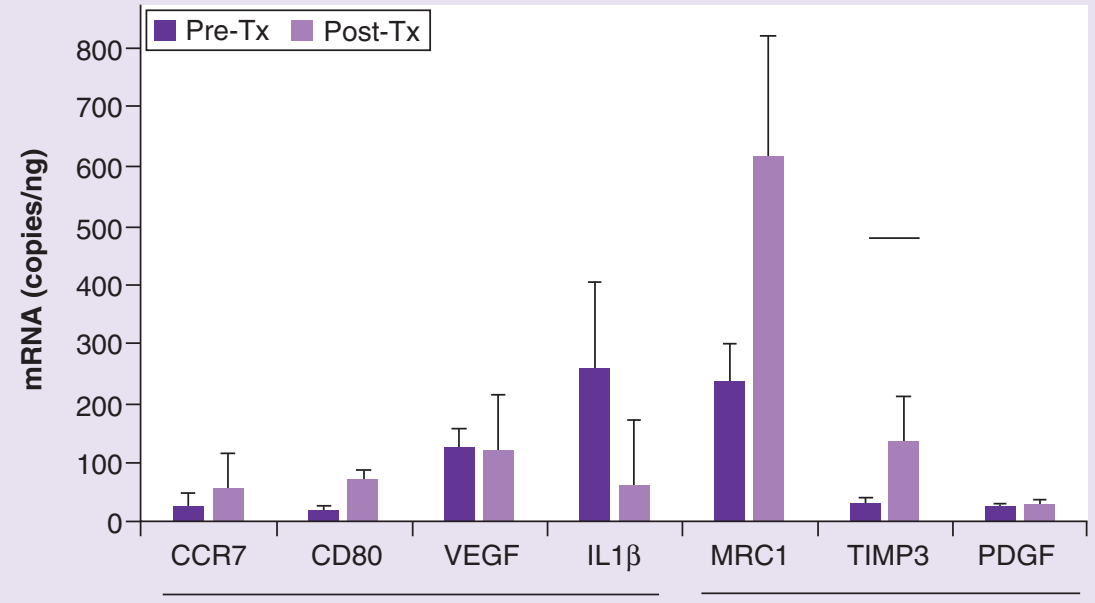

M1

M2

(C)

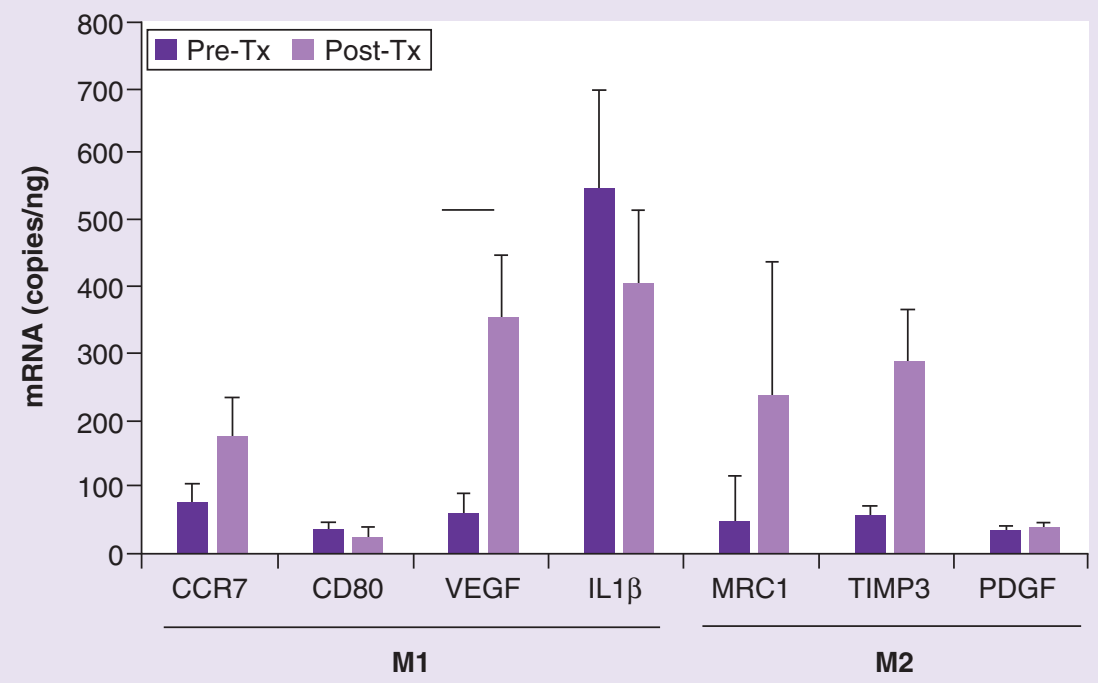


(A)

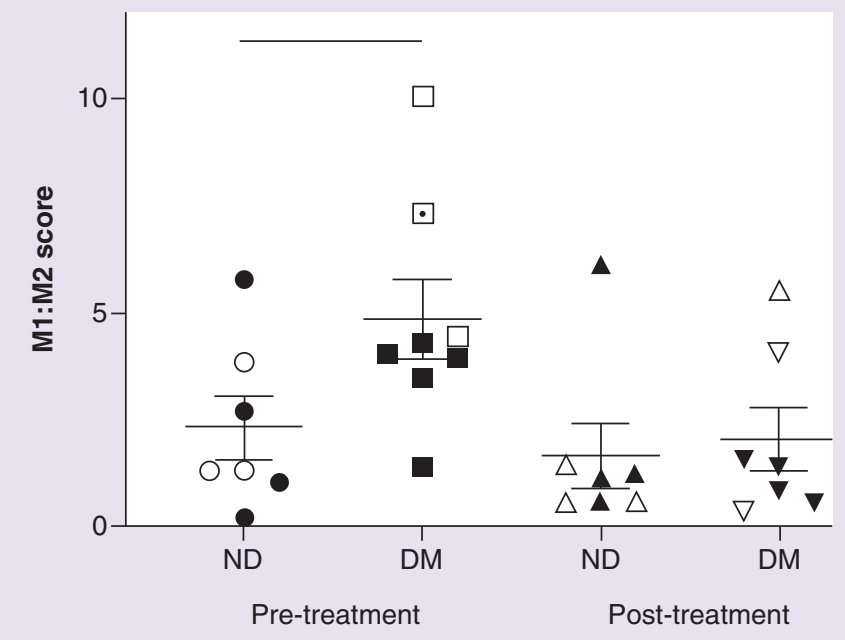

(B)

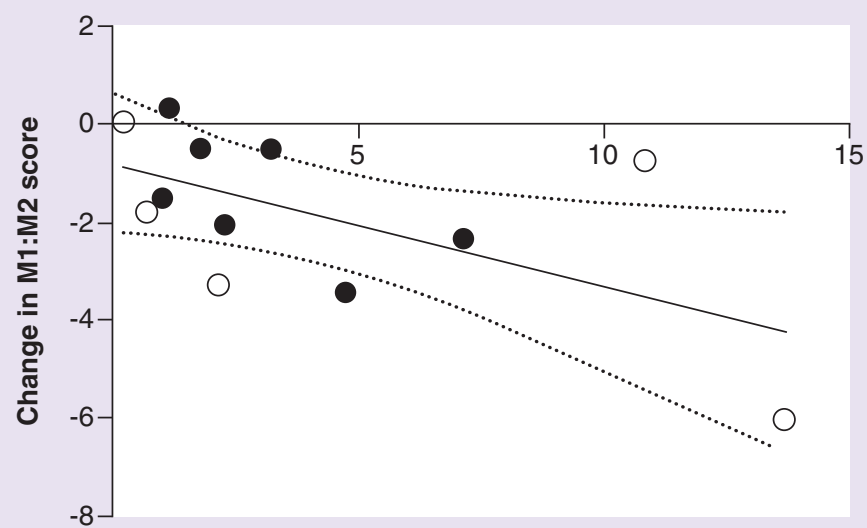

Rate of wound area reduction (\%/day)

Figure 3. Comparison of M1:M2 score between groups and to the rate of wound area reduction. (A) The M1:M2 score in wound debridement samples of diabetic patients is reduced to levels similar to that of nondiabetic patients following treatment with UBM. Bar indicates $\mathrm{p}<0.05$. (B) The M1:M2 score correlates with the rate of wound area reduction. Dotted line indicates the $95 \% \mathrm{Cl}$. Open symbols indicate nonsurgical wounds.

UBM: Urinary bladder matrix.

pro-inflammatory activity. Wounds in diabetic animal models exhibit a prolonged expression of M1 macrophage markers and suppression of M2 macrophage recruitment compare to nondiabetic animal models [18,19]. Multiple mechanisms likely play a role in the increase in M1 macrophage polarization in diabetic patients, including diabetesinduced changes in histone methylation within bone marrow progenitor cells, loss of vascular endothelial growth factor receptor Type 1 signaling, and advanced glycation end-product activation of autophagy [20-22]. More recently, research suggests that the hyperglycemic/diabetic wound environment induces the expression of certain miRNA molecules to favor the inflammatory phenotype in macrophages [23]. Management of wounds with UBM achieved similar macrophage-related gene expression and M1:M2 score in both diabetic and nondiabetic patients despite the diabetic patients initially having a significantly higher M1:M2 score. Therapies that promote increased M2 or inhibit M1 macrophage polarization in the later stages of wound healing are effective at enhancing wound healing in diabetic animal models [24-26]. Similarly, decreases in the M1:M2 score are associated with appropriate wound healing [3,17]. Thus, our data suggest that treatment of diabetic wounds with UBM is associated with a modulation of the inflammation within the wound that enables wound healing.

The mechanism through which UBM facilitates modulation of wound inflammation is likely multifaceted and needs further investigation. Separation of UBM into its soluble and structural fractions identified that the structural components promoted a reduction in macrophage phagocytic activity and an M2-like phenotype [27]. Seeding of macrophages onto UBM results in M2 polarization through activation of peroxisome proliferator activated receptor gamma signaling [28]. Dearth et al. demonstrated that inhibition of cyclooxygenases 1 and 2 reduced M2 polarization and inhibited the accelerated wound healing associated with UBM treatment [29]. A better understanding of these mechanisms and which ones predominate in the diabetic setting will allow for a better design of therapies to promote diabetic wound healing.

Diabetic patients are at high risk for chronic wounds, especially diabetic foot ulcers which have a worldwide prevalence of $6.3 \%$ [30]. In addition to a predilection for developing DFUs, diabetic patients are prone to developing other types of wounds that, due to impaired cellular responses to healing, can develop into chronic conditions, increasing costs. For example, Martin et al. demonstrated in a systematic review that diabetic patients have an associated higher risk of developing surgical site infections [31]. We designed this study to examine whether treatment with UBM would be associated with a restoration of the normal (nondiabetic) state of inflammation in the variety of wounds prone to poor healing in the diabetic population. UBM is a medical device that falls into the skin substitute category from an outpatient reimbursement perspective. To date, applications of UBM in the clinical 
domain are wide ranging, including management of burns, coverage of fasciocutaneous donor sites and as a scaffold for management of full-thickness traumatic wounds with exposed tendons or open fractures [32-37]. Most recently, a randomized controlled trial compared its use in treatment of DFU with standard therapy, suggesting it might have benefit in time-to-healing and reduction in recurrence [13]. Our study provides additional evidence that following treatment with UBM wounds exhibit a reduction in the level of inflammation in the wound, exhibited here by a normalization of the M1:M2 score, thereby resetting the wound environment to the 'wild-type' nondiabetic phenotype.

This study was designed to test whether treatment with UBM was associated with a restoration of a normal inflammatory state in diabetic wounds, and we acknowledge the limitations of this study. Although UBM treatment was associated with a decrease in the M1:M2 score, without longitudinal M1:M2 score data from untreated patients, we cannot rule out that the standard of care would result in a decrease in the M1:M2 score as well. Patients were not randomized nor controlled when selected for this study. We therefore cannot exclude the possibility of bias. No significant differences, however, were found in age, BMI, initial wound area or smoking status between the diabetic and nondiabetic groups, which are all known to be factors that affect wound healing. This study aimed to examine UBM treatment in the multiple types of wounds that exhibit poor healing in the diabetic population and thus included a variety of wound types. The heterogeneity of patients in this study and the variety of treatments (some required different graft sizes) inherent to wound healing studies could have had an influence on results; however, no association between the results and the etiology of the wound were observed. Thus, future studies are needed to examine UBM treatment in specific wound types. It is important to note that other cells in the wound environment may also express these genes, and so the M1:M2 score is more likely an indicator of the character of inflammation as opposed to macrophage phenotype per se. Finally, the small sample size of both groups (nine patients in each) is a limitation, as results could be skewed in one direction. Thus, further work with larger sample sizes, extended treatment times and comparisons to additional treatments are needed in order to fully elucidate the effects of UBM in the diabetic setting.

\section{Conclusion}

UBM has the potential to restore an appropriate inflammatory state in the wounds of diabetic patients, which may facilitate a normal healing response. Treatment with UBM decreased M1:M2 scores, achieving a statistically similar score between diabetic and nondiabetic subjects post-treatment. With modifiable risk factors addressed, this study suggests that UBM may decrease time to healing in diabetic patients by allowing for mitigation of the inflammatory effects of the disease. Future studies in a large, randomized controlled trial may better clarify the abilities of UBM and its efficacy in wound healing and wound care management, especially in the setting of diabetic wounds.

\section{Translational perspective}

Impaired wound healing is common in diabetic patients, increasing the costs associated with the treatment of chronic wounds in this population. Several clinical studies report the use of UBM for the management of chronic wounds in diabetic patients has the potential to improve the time to healing and rates of recurrence [13-16]. Whether this represents a general effect of exposure to UBM common to all patients or represents a specific advantage of the use of UBM in the diabetic population remains unclear. This report demonstrates for the first time that although the wounds of diabetic patients exhibit an elevated M1:M2 score prior to treatment, application of UBM is associated with a restoration of an M1:M2 score similar to that of nondiabetic patients. Such insight provides a scientific basis for the use of UBM in the diabetic population. Future studies are needed to elucidate potential advantages in specific wound types and the effects of long-term application.

Financial \& competing interests disclosure

JT Paige and TC Woods received an investigator-initiated grant support from ACell, Inc. for this study. ACell, Inc. is the manufacturer of the urinary bladder matrix products used in this study. This study was also supported by the NIH through the National Heart Lung \& Blood Institute (R01HL127092/Woods) and the National Institutes of General Medical Sciences (P30GM103337). The authors have no other relevant affiliations or financial involvement with any organization or entity with a financial interest in or financial conflict with the subject matter or materials discussed in the manuscript apart from those disclosed.

No writing assistance was utilized in the production of this manuscript. 


\section{Ethical conduct of research}

The authors state that they have obtained appropriate institutional review board approval or have followed the principles outlined in the Declaration of Helsinki for all human or animal experimental investigations. In addition, for investigations involving human subjects, informed consent has been obtained from the participants involved.

\section{Open access}

This work is licensed under the Attribution-NonCommercial-NoDerivatives 4.0 Unported License. To view a copy of this license, visit http://creativecommons.org/licenses/by-nc-nd/4.0/

\section{Executive summary}

- Application of urinary bladder matrix (UBM) was associated with significant wound size reduction in both diabetic and nondiabetic patients. UBM application was associated with significant increases in the expression of genes associated with the M1 macrophage phenotype, VEGF and the M2 macrophage phenotype, MRC1 and TIMP3.

- A M1:M2 score was calculated based on a set of mRNAs associated with the M1 (IL1 $\beta, C D 80, C C R 7$ and VEGF) and M2 (PDGF, MRC1 and TIMP3) phenotypes.

- Increased rates of wound closure during treatment correlated with decreases in the M1:M2 score pre- versus post-treatment.

- The diabetic patients in this study exhibited a significantly higher M1:M2 score than nondiabetic patients prior to treatment.

- Following treatment with UBM, the M1:M2 scores in the diabetic patients was similar to that of the nondiabetic patients.

- These data suggest that application of UBM is associated with restoration of an appropriate immune response in the wounds of diabetic patients, which may facilitate a normal healing response.

\section{References}

1. American Diabetes Association. Economic costs of diabetes in the U.S. in 2012. Diabetes Care 36(4), 1033-1046 (2013).

2. Das A, Sinha M, Datta $S$ et al. Monocyte and macrophage plasticity in tissue repair and regeneration. Am. J. Pathol. 185(10), 2596-2606 (2015).

3. Nassiri S, Zakeri I, Weingarten MS, Spiller KL. Relative expression of proinflammatory and antiinflammatory genes reveals differences between healing and nonhealing human chronic diabetic foot ulcers. J. Invest. Dermatol. 135(6), 1700-1703 (2015).

4. Fadini GP, De Kreutzenberg SV, Boscaro E et al. An unbalanced monocyte polarisation in peripheral blood and bone marrow of patients with Type 2 diabetes has an impact on microangiopathy. Diabetologia 56(8), 1856-1866 (2013).

5. Badylak SF, Valentin JE, Ravindra AK, McCabe GP, Stewart-Akers AM. Macrophage phenotype as a determinant of biologic scaffold remodeling. Tissue Eng. Part A 14(11), 1835-1842 (2008).

6. Brown BN, Londono R, Tottey $S$ et al. Macrophage phenotype as a predictor of constructive remodeling following the implantation of biologically derived surgical mesh materials. Acta Biomater. 8(3), 978-987 (2012).

7. Brown BN, Valentin JE, Stewart-Akers AM, McCabe GP, Badylak SF. Macrophage phenotype and remodeling outcomes in response to biologic scaffolds with and without a cellular component. Biomaterials 30(8), 1482-1491 (2009).

8. Revi D, Vineetha VP, Muhamed J et al. Wound healing potential of scaffolds prepared from porcine jejunum and urinary bladder by a nondetergent/enzymatic method. J. Biomater. Appl. 29(9), 1218-1229 (2015).

9. Eweida A, Saad M, Gabr E, Marei M, Khalil MR. Cultured keratinocytes on urinary bladder matrix scaffolds increase angiogenesis and help in rapid healing of wounds. Adv. Skin Wound Care 24(6), 268-273 (2011).

10. Beattie AJ, Gilbert TW, Guyot JP, Yates AJ, Badylak SF. Chemoattraction of progenitor cells by remodeling extracellular matrix scaffolds. Tissue Eng. Part A 15(5), 1119-1125 (2009).

11. Dziki JL, Wang DS, Pineda C, Sicari BM, Rausch T, Badylak SF. Solubilized extracellular matrix bioscaffolds derived from diverse source tissues differentially influence macrophage phenotype. J. Biomed. Mater. Res. A 105(1), 138-147 (2017).

12. Sadtler K, Sommerfeld SD, Wolf MT et al. Proteomic composition and immunomodulatory properties of urinary bladder matrix scaffolds in homeostasis and injury. Semin. Immunol. doi:10.1016/j.smim.2017.05.002 (2017) (Epub ahead of print).

13. Alvarez OM, Smith T, Gilbert TW et al. Diabetic foot ulcers treated with porcine urinary bladder extracellular matrix and total contact cast: interim analysis of a randomized, controlled trial. Wounds 29(5), 140-146 (2017).

14. Frykberg RG, Cazzell SM, Arroyo-Rivera J et al. Evaluation of tissue engineering products for the management of neuropathic diabetic foot ulcers: an interim analysis. J. Wound Care 25(Suppl. 7), S18-S25 (2016). 
15. Kimmel H, Rahn M, Gilbert TW. The clinical effectiveness in wound healing with extracellular matrix derived from porcine urinary bladder matrix: a case series on severe chronic wounds. J. Am. Col. Certif. Wound Spec. 2(3), 55-59 (2010).

16. Martinson M, Martinson N. A comparative analysis of skin substitutes used in the management of diabetic foot ulcers. J. Wound Care 25(Suppl. 10), S8-S17 (2016).

17. Bajpai A, Nadkarni S, Neidrauer M, Weingarten MS, Lewin PA, Spiller KL. Effects of non-thermal, non-cavitational ultrasound exposure on human diabetic ulcer healing and inflammatory gene expression in a pilot study. Ultrasound Med. Biol. 44(9), 2043-2049 (2018).

18. Bannon P, Wood S, Restivo T, Campbell L, Hardman MJ, Mace KA. Diabetes induces stable intrinsic changes to myeloid cells that contribute to chronic inflammation during wound healing in mice. Dis. Model Mech. 6(6), 1434-1447 (2013).

19. Okizaki S, Ito Y, Hosono $\mathrm{K}$ et al. Suppressed recruitment of alternatively activated macrophages reduces TGF-beta1 and impairs wound healing in streptozotocin-induced diabetic mice. Biomed. Pharmacother. 70, 317-325 (2015).

20. Gallagher KA, Joshi A, Carson WF et al. Epigenetic changes in bone marrow progenitor cells influence the inflammatory phenotype and alter wound healing in Type 2 diabetes. Diabetes 64(4), 1420-1430 (2015).

21. Okizaki S, Ito Y, Hosono $\mathrm{K}$ et al. Vascular endothelial growth factor receptor Type 1 signaling prevents delayed wound healing in diabetes by attenuating the production of IL-1beta by recruited macrophages. Am. J. Pathol. 186(6), 1481-1498 (2016).

22. Guo Y, Lin C, Xu P et al. AGEs induced autophagy impairs cutaneous wound healing via stimulating macrophage polarization to M1 in diabetes. Sci. Rep. 6, 36416 (2016).

23. Davis FM, Kimball A, Boniakowski A, Gallagher K. Dysfunctional wound healing in diabetic foot ulcers: new crossroads. Curr. Diab. Rep. 18(1), 2 (2018).

24. Chen S, Shi J, Zhang M et al. Mesenchymal stem cell-laden anti-inflammatory hydrogel enhances diabetic wound healing. Sci. Rep. 5, 18104 (2015).

25. Das S, Singh G, Majid M et al. Syndesome therapeutics for enhancing diabetic wound healing. Adv. Healthc. Mater. 5(17), 2248-2260 (2016).

26. Das S, Majid M, Baker AB. Syndecan-4 enhances PDGF-BB activity in diabetic wound healing. Acta Biomater. 42, 56-65 (2016).

27. Slivka PF, Dearth CL, Keane TJ et al. Fractionation of an ECM hydrogel into structural and soluble components reveals distinctive roles in regulating macrophage behavior. Biomater. Sci. 2(10), 1521-1534 (2014).

28. Bullers SJ, Baker SC, Ingham E, Southgate J. The human tissue-biomaterial interface: a role for PPARgamma-dependent glucocorticoid receptor activation in regulating the $\mathrm{CD}_{163}{ }^{+} \mathrm{M} 2$ macrophage phenotype. Tissue Eng. Part A 20(17-18), 2390-2401 (2014).

29. Dearth CL, Slivka PF, Stewart SA et al. Inhibition of COX1/2 alters the host response and reduces ECM scaffold mediated constructive tissue remodeling in a rodent model of skeletal muscle injury. Acta Biomater. 31, 50-60 (2016).

30. Zhang P, Lu J, Jing Y, Tang S, Zhu D, Bi Y. Global epidemiology of diabetic foot ulceration: a systematic review and meta-analysis dagger. Ann. Med. 49(2), 106-116 (2017).

31. Martin ET, Kaye KS, Knott C et al. Diabetes and risk of surgical site infection: a systematic review and meta-analysis. Infect. Control Hosp. Epidemiol. 37(1), 88-99 (2016).

32. Geiger SE, Deigni OA, Watson JT, Kraemer BA. Management of open distal lower extremity wounds with exposed tendons using porcine urinary bladder matrix. Wounds 28(9), 306-316 (2016).

33. Kim JS, Kaminsky AJ, Summitt JB, Thayer WP. New innovations for deep partial-thickness burn treatment with ACell MatriStem Matrix. Adv. Wound Care (New Rochelle) 5(12), 546-552 (2016).

34. Kraemer BA, Geiger SE, Deigni OA, Watson JT. Management of open lower extremity wounds with concomitant fracture using a porcine urinary bladder matrix. Wounds 28(11), 387-394 (2016).

35. Melville JC, Bennetts NA, Tijerina L, Shum JW. The use of acellular urinary bladder matrix as coverage for fasciocutaneous free flap donor sites: an alternative to traditional grafting procedures. J. Oral Maxillofac. Surg. 75 (10), 2254-2260 (2017).

36. Puckett $Y$, Pham T, McReynolds S, Ronaghan C. Porcine urinary bladder matrix for management of infected radiation mastectomy wound. Cureus 9(7), e1451 (2017).

37. Valerio IL, Campbell P, Sabino J, Dearth CL, Fleming M. The use of urinary bladder matrix in the treatment of trauma and combat casualty wound care. Regen. Med. 10(5), 611-622 (2015). 
